\title{
Pulsus paradoxus
}

\author{
Olfa Hamzaoui ${ }^{1}$, Xavier Monnet ${ }^{2,3}$ and Jean-Louis Teboul ${ }^{2,3}$
}

\author{
Number 4 in the Series "Physiology in Respiratory Medicine" \\ Edited by R. Naeije, D. Chemla, A. Vonk-Noordegraaf and A.T. Dinh-Xuan
}

Affiliations: 'Service de Réanimation Médicale, Hôpitaux Universitaires Paris-Sud, Hôpital Antoine Béclère, Clamart, ${ }^{2}$ Service de Réanimation Médicale, Hôpitaux Universitaires Paris-Sud, Hôpital de Bicêtre, Le KremlinBicêtre, ${ }^{3}$ Faculté de Médecine Paris-Sud, Université Paris-Sud, Le Kremlin-Bicêtre, France.

Correspondence: J-L. Teboul, Service de Réanimation Médicale, Centre Hospitalier Universitaire de Bicêtre, 78, Rue du Général Leclerc, 94270 Le Kremlin-Bicêtre, France. E-mail: jean-louis.teboulabct.aphp.fr

ABSTRACT Systolic blood pressure normally falls during quiet inspiration in normal individuals. Pulsus paradoxus is defined as a fall of systolic blood pressure of $>10 \mathrm{mmHg}$ during the inspiratory phase. Pulsus paradoxus can be observed in cardiac tamponade and in conditions where intrathoracic pressure swings are exaggerated or the right ventricle is distended, such as severe acute asthma or exacerbations of chronic obstructive pulmonary disease. Both the inspiratory decrease in left ventricular stroke volume and the passive transmission to the arterial tree of the inspiratory decrease in intrathoracic pressure contribute to the occurrence of pulsus paradoxus. During cardiac tamponade and acute asthma, biventricular interdependence (series and parallel) plays an important role in the inspiratory decrease in left ventricular stroke volume.

Early recognition of pulsus paradoxus in the emergency room can help to diagnose rapidly cardiac tamponade. Measurement of pulsus paradoxus is also useful to assess the severity of acute asthma as well as its response to therapy. Recent development of noninvasive devices capable of automatic calculation and display of arterial pressure variation or derived indices should help improve the assessment of pulsus paradoxus at the bedside.

0 @ERSpublications

A discussion of the possible mechanisms and clinical implications of pulsus paradoxus http://ow.ly/ p7Flt

Previous articles in this series: No. 1: Naeije R, Vachiery J-L, Yerly P, et al. The transpulmonary pressure gradient for the diagnosis of pulmonary vascular diseases. Eur Respir J 2013; 41: 217-223; No. 2: Hughes JMB, van der Lee I. The TL,NO/ $T \mathrm{~L}, \mathrm{CO}$ ratio in pulmonary function test interpretation. Eur Respir J 2013; 41: 453-461. No. 3: Vonk-Noordegraaf A, Westerhof N. Describing right ventricular function Eur Respir J 2013; 41: 1419-1423.

Received: Sept 022012 | Accepted after revision: Nov 212012 | First published online: Dec 062012

Conflict of interest: None declared.

Copyright @ERS 2013 


\section{Introduction}

The current definition of pulsus paradoxus is a fall of systolic blood pressure of $>10 \mathrm{mmHg}$ during the inspiratory phase [1] (fig. 1). The paradox described by Adolf Kussmaul in 1873 was a "pulse simultaneously slight and irregular, disappearing during inspiration and returning upon expiration", despite the continued presence of the cardiac impulse during both respiratory phases.

Pulsus paradoxus can be observed in cardiac tamponade and in conditions where intrathoracic pressure swings are exaggerated or the right ventricle is distended, such as severe acute asthma or exacerbations of chronic obstructive pulmonary disease (COPD).

There is no consensus on the underlying mechanism of pulsus paradoxus. It is likely that several different mechanisms can contribute to the occurrence of pulsus paradoxus. Their respective importance depends on the underlying aetiology (table 1).

\section{Heart-lung interactions during spontaneous breathing}

To understand the complex mechanisms of pulsus paradoxus, we review first the basic principles of heartlung interactions during spontaneous breathing.

Spontaneous inspiration is associated with decrease in intrathoracic pressure (ITP), increase in abdominal pressure and increase in lung volume, which all interfere with circulation in a complex manner. Another important mechanism, which can play a role in the heart-lung interactions, is the biventricular interdependence phenomenon.

\section{Inspiratory decrease in ITP}

Effects on systemic venous return

Because the right atrium is located within the thorax, inspiration leads to a decrease in right atrial pressure relative to atmosphere. As right atrial pressure is the backpressure to systemic venous return, this should result in augmentation of the pressure gradient of systemic venous return (i.e. mean systemic pressure minus right atrial pressure) and accelerate blood flow toward the right heart $[2,3]$. In normal conditions and considering the steep part of the Frank-Starling relationship (stroke volume versus ventricular preload), an increase in ventricular preload is associated with an increase in stroke volume (so-called preloaddependency condition). This latter phenomenon explains the increase in right ventricular stroke volume (RVSV) and pulmonary arterial flow $[2,4]$ that occur at inspiration in normal conditions. However, the augmentation of systemic venous return has its limitation: if right atrial pressure decreases below the atmospheric pressure, the systemic veins collapse as they enter the thorax, thus limiting blood flow [5]. It has to be noted that in case of prior right ventricular dilation or dysfunction, the right ventricle can operate on the flat part of its Frank-Starling curve, so that the increase in right ventricular preload would not induce an increase in RVSV (preload-independency condition).

\section{Effects on right ventricular afterload}

The negative ITP at inspiration may also impede right ventricular afterload. Indeed, the pressure surrounding the right ventricle (i.e. ITP) decreases while the pressure surrounding the intra-alveolar vessels

FIGURE 1 Recording of arterial pressure measured using an arterial catheter in a patient with acute exacerbation of airway obstruction. Pulsus paradoxus is present as the decrease in systolic arterial pressure is $>10 \mathrm{mmHg}$. Note that the arterial pulse pressure also decreases at inspiration, suggesting that left ventricular stroke volume decreases at inspiration. The grey areas indicate the inspiratory phases (Insp). The arrow indicates the amplitude of pulsus paradoxus in this example $(\sim 17 \mathrm{mmHg})$.

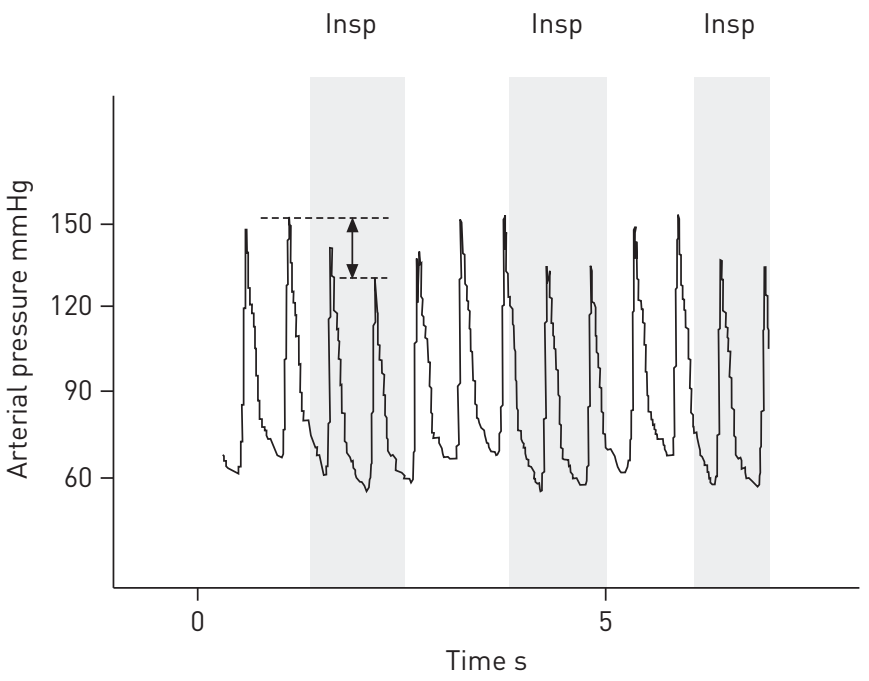


TABLE 1 Main mechanisms contributing to the decrease in systolic blood pressure during inspiration

\begin{tabular}{lcc} 
Mechanisms & Normal subjects & Cardiac tamponade \\
\hline $\begin{array}{l}\text { Passive transmission of decreased intrathoracic pressure } \\
\text { during inspiration }\end{array}$ & + & + \\
$\begin{array}{l}\text { Blood pooling in the pulmonary veins during inspiration } \\
\text { Series biventricular interdependence (pulmonary transit time) }\end{array}$ & $+/-$ & $+/-$ \\
$\begin{array}{l}\text { Parallel biventricular interdependence (septum and } \\
\text { pericardium) }\end{array}$ & 0 & ++ \\
Decreased left ventricular afterload during inspiration & 0 & ++ \\
\hline
\end{tabular}

0 : mechanism is absent; +/-: mechanism may be present or absent; + : mechanism is present and has a moderate contribution; ++: mechanism is present and has an important contribution; +++: mechanism is present and has a very important contribution (a dominant mechanism).

(i.e. alveolar pressure) is close to atmospheric pressure at end-inspiration. Therefore, the right ventricle, which must eject blood into the alveolar arterial bed, has to generate a higher pressure (i.e. transmural pressure) before blood can reach the alveolar vessels. Thus, the right ventricle senses this condition as increased impedance to its ejection [6]. In fact, this phenomenon is only transient because at the same time, the increased driving pressure between alveolar vessels and left atrium (surrounded by a negative ITP) facilitates blood flow to the left atrium and thus results in a reduction in blood volume and pressure in the alveolar vessels until the initial pressure is restored in this new steady state [6]. It must be remembered that stroke volume is much more sensitive to right ventricular afterload increase than to left ventricular afterload increase in healthy subjects.

\section{Effects on left ventricular afterload}

During inspiration, the pressure surrounding the left ventricle (i.e. ITP) decreases while the pressure surrounding the extrathoracic arterial compartment remains constant. As the left ventricle must eject blood toward the extrathoracic arteries, it must generate a higher pressure (i.e. transmural pressure) before blood can leave the thorax. In other words, lowering ITP would be equivalent to raising the arterial pressure by a similar amount and both conditions are sensed as an increased left ventricular afterload [7]. This hypothesis is well illustrated during a Mueller manoeuvre in healthy volunteers, where simultaneous increases in transmural arterial pressure, in end-systolic and in end-diastolic left ventricular volumes, are observed [8]. Similar effects were reported in cardiac surgery patients [9] and in patients with coronary artery disease [8]. However, reduced left ventricular preload rather than increased left ventricular afterload was found by BRINKER et al. [10] using two-dimensional echocardiography during a Mueller manoeuvre in normal men.

Interestingly, in a series of dog experiments, PETERS et al. [11, 12] demonstrated that a decrease in ITP confined to diastole could diminish the ensuing left ventricular stoke volume (LVSV) through reduction in left ventricular preload (see below) [11] while a decrease in ITP confined to systole could increase left ventricular afterload [12].

In animal experiments, Rовотнам et al. [13] observed that during spontaneous ventilation, $\sim 80 \%$ of the time, the minimal and maximal integrated mitral flows preceded the respective minimal and maximal integrated aortic flows. This suggests the dominance of preload effects on afterload effects in determining LVSV [13].

All these findings underline the difficulty of analysing cardiopulmonary interactions with respiration and partly explain some divergent results found in the literature.

\section{Inspiratory increase in abdominal pressure}

At the right side

During spontaneous inspiration, the abdominal pressure increases due to the active descent of the diaphragm. TAKATA et al. [14] have proposed the concept of abdominal vascular zone conditions, analogous to pulmonary vascular zone conditions. In this connection, when the intravascular status is low, the increased abdominal pressure may collapse the inferior vena cava and hence decreases the systemic venous return from the inferior vena cava. When the intravascular volume is high, no critical closure pressure phenomenon is observed since the pressure surrounding the inferior vena cava does not exceed the intraluminal pressure. In this condition, the increased abdominal pressure raises the gradient for systemic venous return. Thus, the net effect of increased abdominal pressure in presence of normo- or hypervolaemia is an increased systemic venous return. 
At the left side

Increased abdominal pressure during inspiration increases the intraluminal pressure of the abdominal aorta and thus the impedance to left ventricular ejection, independently of the effect of decreased ITP [15].

\section{Inspiratory increase in lung volume}

Effects on right ventricular afterload

The relationship between lung vessels resistance and lung volume is complex.

From a series arrangement viewpoint, the pulmonary circulation may be divided into extra-alveolar vessels and intra-alveolar vessels [6]. Inspiratory increase in lung volume compresses the lumens of intra-alveolar vessels resulting in an exponential increase in intra-alveolar vessels resistance (fig. 2). By contrast, increased lung volume induces an exponential decrease in extra-alveolar vessels resistance from residual volume to total lung capacity (fig. 2). Indeed, as lung volume increases, the radial interstitial forces increase, resulting in the widening of extra-alveolar vessels. Thus, the total pulmonary vascular resistances describe a " $U$ " shape with a nadir corresponding to a lung volume equal to the relaxation volume or functional residual capacity [16] (fig. 2). In healthy subjects, there is a slight increase in pulmonary vascular resistances during tidal inspiration. This effect can be more marked during exacerbations of COPD or asthmatic attacks where the end-expiratory lung volume is significantly higher than the relaxation volume.

From a parallel arrangement viewpoint, the pulmonary circulation is distributed along a gravitational gradient of vascular-alveolar pressure difference [6]. By raising transpulmonary pressure (alveolar pressure minus ITP), inspiration may cause a larger proportion of the pulmonary circulation to behave as West's zone 2, especially when the pulmonary venous pressure is low. This contributes to increased pulmonary vascular resistance and right ventricular afterload, especially in cases of low blood volume states [17].

\section{Effects on left ventricular afterload}

During spontaneous inspiration, a decrease in the left ventricular septal-lateral dimension consistent with compression of the heart in the lateral direction has been reported [18]. However, Scharf et al. [19] showed that the compressive effect of lung inflation probably plays a less important role during spontaneous inspiration than during mechanical ventilation with positive end-expiratory pressure.

\section{Biventricular interdependence}

Biventricular interdependence can occur in a parallel or in a series manner.

\section{Parallel biventricular interdependence}

The two ventricles are surrounded by a poorly expandable membrane, the pericardium. During inspiration, the increase in right ventricular end-diastolic volume increases the surrounding pericardial pressure relative to the ITP. This, in turn, increases the pressure in the left atrium and thus reduces the left atrial filling. In addition, if the right ventricular filling pressure increases enough during inspiration, the septum shifts leftward (fig. 3). This leads to a decreased compliance of the left ventricle, which further limits its filling. The presence of the pericardium markedly enhances the degree of diastolic ventricular interaction [20].

FIGURE 2 Relationships between pulmonary vascular resistances and lung volume (see text for detailed explanations). RV: residual volume; FRC: functional residual capacity; TLC: total lung capacity.

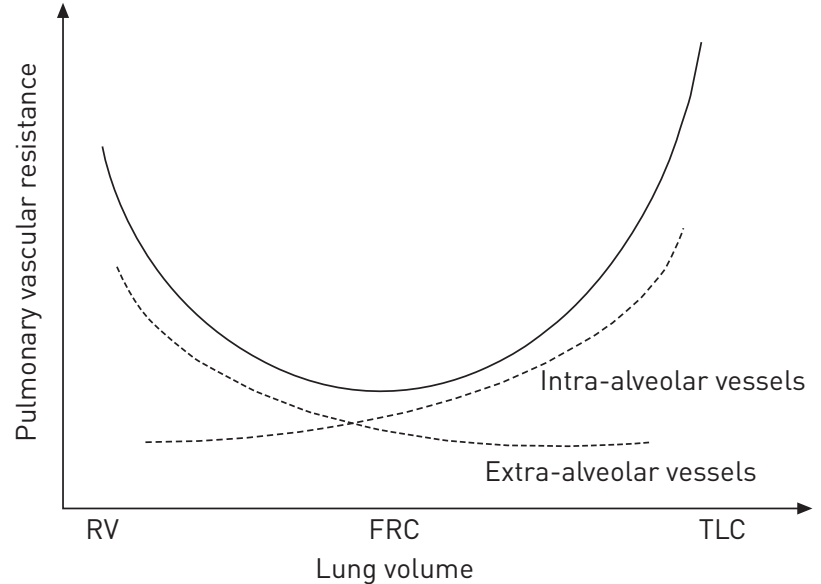




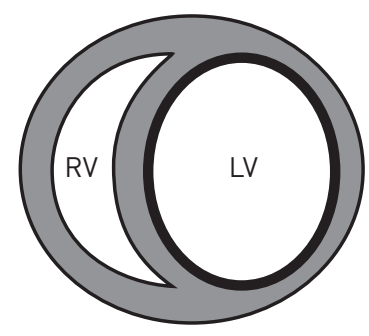

Expiration

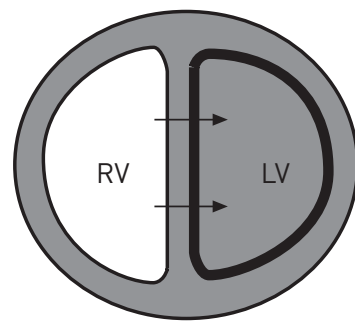

Inspiration
FIGURE 3 Parallel biventricular interdependence. In some pathological conditions, inspiration results in a marked dilation of the right ventricle (RV). Because expansion of the pericardium is limited, this will result in a leftward shift of the interventricular septum (arrows) and thus in impediment of left ventricle (LV) filling. This, in turn, results in a decrease in the left ventricular stroke volume during inspiration.

\section{Series biventricular interdependence}

Blood pumped from the right ventricle travels through the pulmonary circulation to the left atrium. Thus, respiration-induced changes in RVSV affect left ventricle filling. Because the pulmonary transit time is long (several seconds), the stroke volume, which is maximal during inspiration at the output of the right ventricle, can be maximal during expiration at the output of the left ventricle, and vice versa. It is likely that the series ventricular interaction, which is operating in case of biventricular preload-dependency (fig. 4), plays a role in the changes in pulse pressure and LVSV variation observed during quiet spontaneous breathing in normal subjects [21]. However, this could be different in cases of low respiratory rate or change in the inspiratory-to-expiratory time ratio. In addition, there is a great variability of the pulmonary transit time even in normal subjects at rest (between 5 and $10 \mathrm{~s}$ ) [22]. The pulmonary transit time decreases when cardiac output increases, and reaches its minimum value when cardiac index is $\sim 8 \mathrm{~L} \cdot \mathrm{min}^{-1} \cdot \mathrm{m}^{-2}$ [22]. The pulmonary transit time can be increased in congestive heart failure [23] or pulmonary hypertension [24], independently of the value of cardiac output. For all these reasons, it is difficult to predict which role is played by series ventricular interaction in the occurrence of LVSV respiratory variation. When the two ventricles are $180^{\circ}$ out of respiratory phase, this can enhance the degree of LVSV variation. When the two ventricles are in phase, the LVSV variation due to other mechanisms could be attenuated or even absent.

\section{Mechanisms of pulsus paradoxus}

Respiratory changes in arterial pressure during normal breathing conditions

In healthy subjects, quiet inspiration results in a decrease in systolic arterial pressure [25], which can be related to either the direct transmission to the arterial tree of the decreased ITP [26] or to the decrease in LVSV [27-30], or, more probably, both. As detailed above, the inspiratory decrease in LVSV, which is associated with inspiratory increases in vena caval flow $[3,31]$, right ventricular end-diastolic volume, RVSV and pulmonary arterial flow $[2,3,29]$, can be the result of decrease in left ventricular preload or left ventricular afterload, or both. It is unlikely that the small decrease in ITP during quiet inspiration in healthy

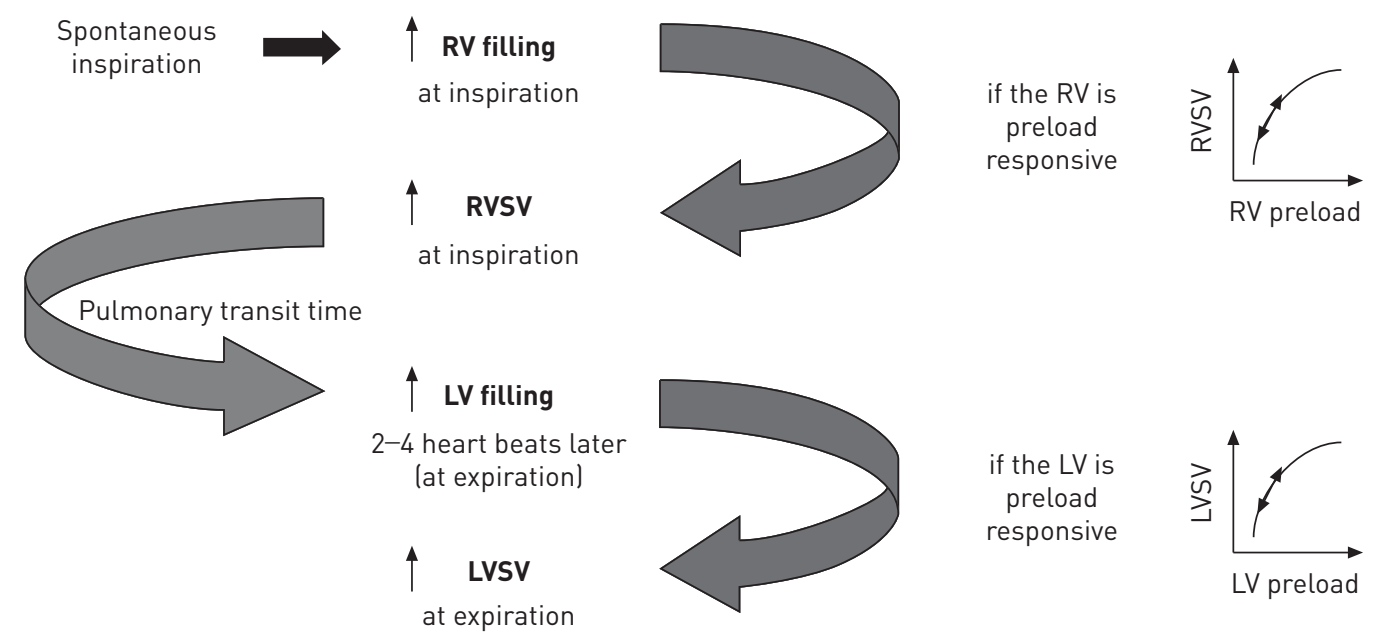

FIGURE 4 Series biventricular interdependence. Inspiration results in increase in filling and stroke volume (SV) of the right ventricle $(\mathrm{RV})$. Because of the long pulmonary transit time, there is a phase lag between the right ventricle and the left ventricle (LV) so that LVSV is maximal during expiration. All these mechanisms are fully operating in cases of biventricular preload-dependency (i.e. RV and LV operating on the steep part of their Frank-Starling curve). 
individuals $(\sim 5 \mathrm{mmHg})$ can result in a significant decrease in LVSV related to increased left ventricular afterload. In this regard, left ventricular end-diastolic volume was shown to decrease rather than to increase during inspiration $[27,28]$. The reduction in left ventricular preload during inspiration, which is thus the most plausible explanation, could be due: either to 1) series ventricular interaction (see above), making the right and left ventricles $180^{\circ}$ out of phase; 2) reduction in left ventricular diastolic time related to the increased heart rate accompanying inspiration; or 3) the parallel ventricular interdependence phenomenon. However, it is unlikely that the latter mechanism is significant in the absence of pericardial constraint or of right ventricle overdistension during normal inspiration [32]. Another possible mechanism could be an inspiratory pulmonary venous blood pooling, related to the fact that pulmonary vessels are supposed to be more compliant than the left ventricle $[25,33]$.

Since in physiological conditions, the inspiratory decrease in ITP is low $(<5 \mathrm{mmHg})$ and the inspiratory decrease in LVSV is small $(<10 \%)[4,25]$, the inspiratory decrease in systolic arterial pressure is physiologically $<10 \mathrm{mmHg}(\sim 5 \mathrm{mmHg})[25]$.

\section{Pulsus paradoxus in cardiac tamponade}

Cardiac tamponade is a pathological condition where fluid accumulation in the pericardial sac surrounding the heart causes elevation and equilibration of pericardial and cardiac chamber pressures, reduced cardiac output, and pulsus paradoxus.

Different mechanisms leading to pulsus paradoxus (table 1) can be involved.

In this situation, ITP decreases during inspiration less than in acute asthma but probably more than in normal conditions. Because the right atrial pressure is high at expiration, the decrease in ITP should further increase more the inferior vena cava flow than in normal conditions as the inferior vena cava flow limitation is attenuated. The large inspiratory increases in vena cava flow [31,34] and right ventricle filling should result in a large inspiratory increase in RVSV, as confirmed by experimental $[2,34]$ and clinical studies $[4,25]$. In an experimental model of cardiac tamponade, SHABETAI et al. [31] found that when venous return to the right atrium was held constant throughout the respiratory cycle (right heart bypass), pulsus paradoxus no longer appeared. This confirms the important role of biventricular interdependence (parallel and/or series) in the development of pulsus paradoxus. Unlike normal conditions, cardiac tamponade should be associated with a marked parallel biventricular interaction because of space limitations imposed by the pericardial constraint. In accordance with this potential mechanism, SETTLE et al. [35], using echocardiography, reported increased right ventricular dimensions and decreased left ventricular dimensions during inspiration. In each instance, this was caused by a posterior movement of the interventricular septum toward the left ventricle in inspiration. Both parallel and series biventricular interdependence mechanisms should contribute to decrease left ventricle filling and hence to decrease LVSV at inspiration if the left ventricle is preload-dependent. RAMACHANDRAN et al. [36] used a human cardiovascular-respiratory system model that simulates haemodynamic and respiratory changes associated with tamponade clinically. They showed that both the parallel and series interactions have an equal and significant contribution to LVSV respiratory variation, and hence to pulsus paradoxus.

\section{Pulsus paradoxus in acute asthma}

The presence of large inspiratory decreases in ITP and breathing at high lung volume are the main characteristics explaining the occurrence of pulsus paradoxus during acute asthma.

The large decrease in ITP during inspiration $(-30--20 \mathrm{mmHg})$ [37] can affect systolic arterial pressure through both direct (passive transmission along the arterial tree) and indirect (decrease in LVSV) mechanisms. JARDIN et al. [37], studying nine patients with acute asthma, reported a mean inspiratory decrease in systolic arterial pressure of $41 \mathrm{mmHg}$, in arterial pulse pressure of $23 \mathrm{mmHg}$ and in diastolic arterial pressure of $18 \mathrm{mmHg}$. The decrease in pulse pressure suggests that LVSV decreases at inspiration while the large decrease in diastolic pressure suggests that passive transmission of ITP along the arterial tree does not play a minor role.

It is far from certain that pulsus paradoxus in acute asthma is only the exaggeration of the inspiratory decrease in systolic arterial pressure in healthy subjects. In both conditions, the right atrial pressure is generally not high at expiration. As the inferior vena cava flow is limited during deep inspiration when the right atrial pressure falls below the atmospheric pressure, the increase in systemic venous return is thus not exaggerated. At the same time, right ventricular afterload increases because of both the deep decrease in ITP (see above) and the effect of lung inflation on the pulmonary vascular resistance. As detailed above, in acute asthma where functional residual capacity is markedly increased [38], the inspiratory increase in lung volume should result in an increased pulmonary vascular resistance. The ensuing increase in right ventricular afterload was well illustrated in seven patients with acute asthmatic attack [39]. Using 
two-dimensional echocardiography examination in the short axis, JARDIN et al. [40] measured significant increases in right ventricular end-systolic and end-diastolic areas at inspiration [38], findings that were not reported in healthy volunteers. Moreover, right ventricular stroke area and pulmonary artery pulse pressure decrease during inspiration, findings that are the opposite of what is expected in normal subjects [4]. However, divergent findings were reported in a clinical study including asthmatic patients with cold air bronchial hyperreactivity before and during a bronchial challenge with cold air mimicking an asthmatic attack [41]. In this study, right ventricle and left ventricle volumes and ejection fractions were measured using radionuclide angiography during inspiration and expiration [41]. The bronchial challenge produced pulsus paradoxus and changes in oesophageal pressure (measuring ITP) that were significantly greater than those during quiet breathing [41]. These changes were accompanied by a decrease in left ventricle diastolic volume and in LVSV during inspiration and an increase in these variables during expiration; right ventricular diastolic volume and RVSV demonstrated changes reciprocal to those seen in the left ventricle [40]. Although these two clinical studies [39, 41] found divergent results in terms of RVSV during inspiration, both reported a marked inspiratory increase in right ventricular end-diastolic volume and suggested that this mechanism is responsible for the occurrence of pulsus paradoxus. It is thus likely that in acute asthma, the increased systemic venous return and, presumably, the increased right ventricular afterload during inspiration result in an inspiratory right ventricle overdistension $[39,41]$ and thus in a reduced left ventricle filling $[37,41]$ at the same time through parallel biventricular interdependence. If the left ventricle is preload-dependent, this could result in a decrease in LVSV at inspiration [37, 41]. Other mechanisms of pulsus paradoxus are shown in table 1.

Inspiratory increases in left ventricle afterload could play a role. Although at the right side, a marked fall in ITP does not induce an exaggerated increase in right ventricular preload because of inferior vena cava flow limitation, it can result in a marked increase in left ventricular afterload. Even if it exists, this effect is difficult to identify because of the probable predominant biventricular interdependence effect.

Series biventricular interdependence could play a role in the development of pulsus paradoxus because the two ventricles are $180^{\circ}$ out of phase, if respiratory rate, inspiratory-to-expiratory ratio and pulmonary transit time are normal. This could be different in some instances during acute asthma, for example if the respiratory rate is abnormally low and the pulmonary transit time reduced in relation to a high cardiac output.

Studying patients with airway obstruction during normal respiration, and after a brief period of apnoea, RUSKIN et al. [25] presented arguments against a major role of biventricular interdependence (series or parallel). Indeed, the fact that the inspiratory decrease of LVSV following a period of apnoea was identical in magnitude with that observed during continuous respiration indicated that any contribution to respiratoryrelated alterations of LVSV by preceding alterations of right ventricular output during continuous respiration must have been minimal [25]. They suggested that the inspiratory fall in LVSV during inspiration resulted from transient inspiratory pooling of blood in the pulmonary veins with a resultant decrease in left ventricle filling [25].

Finally, the role of volume depletion was raised by SQUARA et al. [42] in a clinical study performed in 10 patients with acute asthma and pulsus paradoxus. Pulsus paradoxus decreased during military antishock trouser (MAST) inflation and returned to baseline values after MAST deflation [41]. Interpretation of these results is speculative. A first possibility is that MAST inflation made the heart (right ventricle and/or left ventricle) preload-independent such that any change in right ventricular or left ventricular end-diastolic volume no longer resulted in parallel change in RVSV or LVSV. A second possibility is that volume loading reduced the extent of West's zone 2 and, thus, the degree of inspiratory increase in pulmonary vascular resistance and right ventricular afterload and hence the degree of parallel biventricular interdependence. Although this study did not indicate the major mechanism of pulsus paradoxus in acute asthma with certainty, it clearly indicates that the direct and passive transmission of decreased ITP on the arterial system cannot be the sole mechanism.

Given all these divergent findings, it is difficult to propose a unique theory to explain pulsus paradoxus during acute asthma. It is likely that several mechanisms are involved.

\section{Pulsus paradoxus in acute exacerbation of COPD}

Mechanisms of pulsus paradoxus during acute exacerbation of COPD could be a little different from acute asthma. First, unlike in asthma, right atrial pressure is generally high at expiration in COPD patients during acute exacerbation, so that the degree of vena cava flow limitation during the ensuing deep inspiration should be attenuated. In this connection, a large inspiratory right ventricular filling is expected to occur. In addition, lung hyperinflation and gas trapping could result in a positive intrathoracic pressure at expiration, in relation to the development of intrinsic positive end-expiratory positive pressure. This should result in a decrease in systemic venous return and right ventricular preload at expiration, which is expected to be more 
marked in low central venous pressure states [43]. Combination of these two effects (decrease in systemic venous return at expiration and enhanced systemic venous return at inspiration) should result in a marked respiratory change in systemic venous return and RVSV and, thus, in the development of pulsus paradoxus through series biventricular interdependence. In addition, right ventricular afterload should increase during inspiration (breathing at high lung volume). During acute exacerbation of COPD, where the right ventricle can already be enlarged, combination of increased right ventricular preload and increased right ventricular afterload during inspiration should result in a marked parallel biventricular interdependence phenomenon, as demonstrated by SETTLE et al. [32] in an electrocardiographic study. This can contribute to the development of pulsus paradoxus during acute exacerbation of COPD. As in acute asthma, direct transmission of decreased ITP and increased left ventricular afterload during inspiration can play a role in the development of pulsus paradoxus [26].

\section{Pulsus paradoxus in other conditions}

Finally, pulsus paradoxus may rarely be observed in massive pulmonary embolism, large compressive pleural effusion, profound hypovolaemia and tricuspid atresia.

\section{Clinical implications \\ Measurement of pulsus paradoxus}

Pulsus paradoxus is traditionally measured using a sphygmomanometer. The brachial cuff must be inflated above the presumed value of systolic arterial pressure and then deflated slowly to find the highest pressure at which the first Korotkoff sound is heard, normally during expiration. The cuff must be further deflated to the pressure at which the Korotkoff sounds are heard during both inspiration and expiration. Pulsus paradoxus is present if the difference between the latter and the former pressures is $>10 \mathrm{mmHg}$. In general, the procedure is repeated two or three times to improve accuracy. However, this method is cumbersome and time-consuming (2-5 $\mathrm{min}$ ) [44]. In addition, in some patients, audibility of sounds is poor because of tachypnoea and a noisy clinical environment (emergency room). In a great number of cases, experienced physicians have failed to measure reliably the amplitude of pulsus paradoxus [45].

Because of all these drawbacks, $>98 \%$ of care providers do not use the manual measurement at the bedside in acute asthma [46]. Alternative methods have been proposed to measure pulsus paradoxus, in particular methods allowing automatic and real-time measurements, such as noninvasive blood pressure monitors [47] and pulse oximetry [48]. In a series of 26 patients with COPD, respiratory waveform variation of pulse oximetry closely correlated with pulsus paradoxus measured using sphygomanometry [48]. In an intensive care context, invasive monitoring of blood pressure using an arterial catheter provides an accurate real-time assessment of pulsus paradoxus.

\section{Clinical significance of pulsus paradoxus \\ Cardiac tamponade}

GUBERMAN et al. [49] reported that pulsus paradoxus is detectable in $98 \%$ of patients with cardiac tamponade, even in the absence of hypotension. Its early recognition can thus help to reach diagnosis rapidly, which must be confirmed by electrocardiography. After pericardiocentesis, pulsus paradoxus and respiratory variation in transvalvular flow velocities decrease [4].

\section{Acute asthma}

Measurement of pulsus paradoxus during acute asthma exacerbations is currently recommended by international guidelines [50]. It is considered to be a surrogate of airflow obstruction indexes in the emergency ward [51,52] and is a key variable of the severity scores developed in acute asthma [50, 52-54]. Of note, in cases of severe respiratory distress with respiratory exhaustion, swings in ITP can be reduced and pulsus paradoxus can be absent.

Until now, pulsus paradoxus has not been frequently measured because of the cumbersomeness of the manual method. Development of continuous noninvasive methods should make the measurement of pulsus paradoxus in the emergency room more frequent, and thus ease the clinical assessment of severity and response to treatment. In asthmatic patients presenting to the emergency room, arterial tonometry was used for automated measurement of inspiratory decrease in systolic pressure [45]. A value of $11 \mathrm{mmHg}$ measured after $60 \mathrm{~min}$ of standardised asthmatic treatment allowed the discrimination of patients who were eventually discharged from those who were eventually hospitalised (triage use) [45]. Noninvasive finger blood pressure monitors can also assess pulsus paradoxus and its response to treatment [45]. Importantly, a variable derived from pulse oximetry respiratory waveform variation was demonstrated to correlate with airflow obstruction indexes in patients with acute asthma [52]. Incorporation of such variables into pulse oximeters has been proposed to improve the clinical assessment of asthma severity and to facilitate 
following of the response to treatment, particularly in patients who cannot undergo spirometry because of young age or severity of illness [52].

Pulsus paradoxus can be present in other acute or chronic airway obstruction diseases. Pulse oximetry respiratory waveform variation was demonstrated to correlate with the degree of air trapping in patients with COPD [48]. In children who presented to the emergency department with signs of croup, the amplitude of pulsus paradoxus was correlated to the Westley croup score, and the degree of change in this amplitude in response to epinephrine was correlated to the change in the Westley croup score [55]. In obstructive sleep apnoea [56], the presence of pulsus paradoxus is also a marker of severity since it indicates a marked inspiratory decreased ITP. Its disappearance after continuous positive airway pressure application can be an indicator of treatment efficiency [56].

It is noteworthy that respiratory changes in arterial pulse pressure can occur during mechanical ventilation. The so-called pulse pressure variation is considered to be a marker of biventricular preload-dependency and, as such, a reliable indicator of fluid responsiveness in mechanically ventilated patients $[57,58]$. The purpose of the present article is not to review the mechanisms, significance and limits of pulse pressure variation, since they have been extensively detailed in recent literature [58, 59].

\section{Conclusion}

Biventricular interdependence (series and parallel) and passive transmission to the arterial tree of the inspiratory decrease in ITP play an important role in the appearance of pulsus paradoxus during cardiac tamponade and acute asthma. Recent development of noninvasive devices able to automatically calculate and display arterial pressure variation or derived indices should make the assessment of pulsus paradoxus at the bedside easier.

\section{References}

Bilchick KC, Wise RA. Paradoxical physical findings described by Kussmaul's sign. Lancet 2002; 359: 1940-1942. Shabetai R, Fowler NO, Fenton JC, et al. Pulsus paradoxus. J Clin Invest 1965; 44: 1882-1898.

Morgan BC, Martin WE, Hornbein TF, et al. Hemodynamic effects of intermittent positive pressure respiration. Anesthesiology 1966; 27: 584-590.

4 Appleton CP, Hatle LK, Popp RL. Cardiac tamponade and pericardial effusion: respiratory variation in transvalvular flow velocities studied by Doppler echocardiography. J Am Coll Cardiol 1988; 11: 1020-1030.

5 Guyton AC, Adkins LH. Quantitative aspects of the collapse factor in relation to venous return. Am J Physiol 1954; 177: 523-527.

6 Permutt S, Wise RA, Brower RG. How changes in pleural and alveolar pressure cause changes in afterload and preload. In: Scharf SM, Cassidy SS, eds. Heart-Lung Interactions in Health and Disease. New-York, Marcel Dekker, 1989; pp. 243-250.

7 Robotham JL, Mintzner W. A model of the effects of respiration on left ventricular performance. J Appl Physiol 1979; 46: 411-418.

8 Scharf SM, Bianco JA, Tow DE, et al. The effects of large negative intrathoracic pressure on left ventricular function in patients with coronary artery disease. Circulation 1981; 63: 871-875.

9 Buda AJ, Pinsky MR, Ingels NB Jr, et al. Effect of intrathoracic pressure on left ventricular performance. $N$ Engl J Med 1979; 301: 453-459.

10 Brinker JA, Weiss JL, Lappé DL, et al. Leftwards septal displacement during right ventricular loading in man. Circulation 1980; 61: 626-633.

11 Peters J, Kindred MK, Robotham JL. Transient analysis of cardiopulmonary interactions. I. Diastolic events. J Appl Physiol 1988; 64: 1506-1517.

12 Peters J, Kindred MK, Robotham JL. Transient analysis of cardiopulmonary interactions II. Systolic events. J Appl Physiol 1988; 64: 1518-1526.

13 Robotham JL, Stuart RS, Doherty K, et al. Mitral and aortic blood flows during spontaneous respiration in dogs. Anethesiology 1988; 69: 516-526.

14 Takata M, Wise RA, Robotham JL. Effects of abdominal pressure on venous return: abdominal vascular zone conditions. J Appl Physiol 1990; 69: 1961-1972.

15 Robotham JL, Wise RA, Bromberger-Barnea B. Effects of changes in abdominal pressure on left ventricular performance and regional blood flow. Crit Care Med 1985; 13: 803-809.

16 Whittenberger JL, McGregor M, Berglund E, et al. Influence of state of inflation of the lung on pulmonary vascular resistance. J Appl Physiol 1960; 15: 878-882.

17 Fougères E, Teboul JL, Richard C, et al. Hemodynamic impact of a positive end-expiratory pressure setting in acute respiratory distress syndrome: importance of the volume status. Crit Care Med 2010; 38: 802-807.

18 Robotham JL, Badke FR, Kindred MK, et al. Regional left ventricular performance during normal and obstructed spontaneous respiration. J Appl Physiol 1983; 55: 569-577.

19 Scharf SM, Brown R, Warner KG, et al. Intrathoracic pressures and left ventricular configuration with respiratory maneuvers. J Appl Physiol 1989; 66: 481-491.

20 Janicki JS, Weber KT. The pericardium and ventricular interaction, distensibility, and function. Am J Physiol 1980; 238: H494-H503.

21 Guntheroth WC. Sensitivity and specificity of echocardiographic evidence of tamponade: implications for ventricular interdependence and pulsus paradoxus. Pediatr Cardiol 2007; 28: 358-362.

22 Zavorsky GS, Walley KR, Russell JA. Red cell pulmonary transit times through the healthy human lung. Exp Physiol 2003; 88: 191-200. 
23 Shors SM, Cotts WG, Pavlovic-Surjancev B, et al. Heart failure: evaluation of cardiopulmonary transit times with time-resolved MR angiography. Radiology 2003; 229: 743-748.

24 Skrok J, Shehata ML, Mathai S, et al. Pulmonary arterial hypertension: MR imaging-derived first-pass bolus kinetic parameters are biomarkers for pulmonary hemodynamics, cardiac function, and ventricular remodeling. Radiology 2012; 263: 678-687.

25 Ruskin J, Bache RJ, Rembert JC, et al. Pressure-flow studies in man: effect of respiration on left ventricular stroke volume. Circulation 1973; 48: 79-85.

26 Viola AR, Puy RJM, Goldman E. Mechanisms of pulsus paradoxus in airway obstruction. J Appl Physiol 1990; 68: 1927-1931.

27 Brenner JI, Waugh RA. Effect of phasic respiration on left ventricular dimension and performance in a normal population. An echocardiographic study. Circulation 1978; 57: 122-127.

28 Andersen K, Vik-Mo H. Effects of spontaneous respiration on left ventricular function assessed by echocardiography. Circulation 1984; 69: 874-879.

29 Leeman DE, Levine MJ, Come PC. Doppler echocardiography in cardiac tamponade: exaggerated respiratory variation in transvalvular blood flow velocity integrals. J Am Coll Cardiol 1988; 11: 572-578.

30 van den Hout RJ, Lamb HJ, van den Aardweg JG, et al. Real-time MR imaging of aortic flow: influence of breathing on left ventricular stroke volume in chronic obstructive pulmonary disease. Radiology 2003; 229: 513-519.

31 Shabetai R, Fowler NO, Guntheroth WG. The hemodynamics of cardiac tamponade and constrictive pericarditis. Am J Cardiol 1970; 26: 480-489.

32 Settle HP Jr, Engel PJ, Fowler NO, et al. Echocardiographic study of the paradoxical arterial pulse in chronic obstructive lung disease. Circulation 1980; 62: 1297-1307.

33 Lange RL, Tsagaris TJ. Time course of factors causing exaggerated respiratory variation of arterial blood pressure. J Lab Clin Med 1964; 63: 431-444.

34 Guntheroth WG, Morgan BC, Mullins GL. Effect of respiration on venous return and stroke volume in cardiac tamponade. Mechanism of pulsus parodoxus. Circ Res 1967; 20: 381-390.

35 Settle HP, Adolph RJ, Fowler NO, et al. Echocardiographic study of cardiac tamponade. Circulation 1977; 56: 951-959.

36 Ramachandran D, Luo C, Ma TS, et al. Using a human cardiovascular-respiratory model to characterize cardiac tamponade and pulsus paradoxus. Theor Biol Med Model 2009; 6: 15.

37 Jardin F, Farcot JC, Boisante L, et al. Mechanism of paradoxic pulse in bronchial asthma. Circulation 1982; 66: 887-894.

38 Woolcock AJ, Read J. Lung volumes in exacerbations of asthma. Am J Med 1966; 41: 259-273.

39 Jardin F, Dubourg O, Margairaz A, et al. Inspiratory impairment in right ventricular performance during acute asthma. Chest 1987; 92: 789-795.

40 Jardin F, Farcot JC, Guéret P, et al. Echocardiographic evaluation of ventricles during continuous positive airway pressure breathing. J Appl Physiol 1984; 56: 619-627.

41 Blaustein AS, Risser TA, Weiss JW, et al. Mechanisms of pulsus paradoxus during resistive respiratory loading and asthma. J Am Coll Cardiol 1986; 8: 529-536.

42 Squara P, Dhainaut JF, Schremmer B, et al. Decreased paradoxic pulse from increased venous return in severe asthma. Chest 1990; 97: 377-383.

43 Boerrigter B, Trip P, Bogaard HJ, et al. Right atrial pressure affects the interaction between lung mechanics and right ventricular function in spontaneously breathing COPD patients. PLoS One 2012; 7: e30208.

44 Shim C, Williams MH Jr. Pulsus paradoxus in asthma. Lancet 1978; 8063: 530-531.

45 Jay GD, Onuma K, Davis R, et al. Analysis of physician ability in the measurement of pulsus paradoxus by sphygmomanometry. Chest 2000; 118: 348-352.

46 Canny GJ, Reisman J, Healy R, et al. Acute asthma: observations regarding the management of a pediatric emergency room. Pediatrics 1989; 83: 507-512.

47 Rayner J, Trespalacios F, Machan J, et al. Continuous noninvasive measurement of pulsus paradoxus complements medical decision making in assessment of acute asthma severity. Chest 2006; 130: 754-765.

48 Hartert TV, Wheeler AP, Sheller JR. Use of pulse oximetry to recognize severity of airflow obstruction in obstructive airway disease: correlation with pulsus paradoxus. Chest 1999; 115: 475-481.

49 Guberman BA, Fowler NO, Engel PJ, et al. Cardiac tamponade in medical patients. Circulation 1981; 64: 633-640

50 National Heart, Lung, and Blood Institute National Asthma Education and Prevention Program. Expert Panel Report 3: Guidelines for the Diagnosis and Management of Asthma. National Institutes of Health, Bethesda, 2007. www.nhlbi.nih.gov/guidelines/asthma/asthgdln.pdf Date last accessed: XXX. Date last updated: August 28, 2007.

51 Arnold DH, Gebretsadik T, Minton PA, et al. Clinical measures associated with FEV1 in persons with asthma requiring hospital admission. Am J Emerg Med 2007; 25: 425-429.

52 Arnold DH, Jenkins CA, Hartert TV. Noninvasive assessment of asthma severity using pulse oximeter plethysmograph estimate of pulsus paradoxus physiology. BMC Pulm Med 2010; $10: 17$.

53 Fischl MA, Pitchenik A, Gardner LB. An index predicting relapse and need for hospitalization in patients with acute bronchial asthma. N Engl J Med 1981; 305: 783-789.

54 Arnold DH, Gebretsadik T, Minton PA, et al. Assessment of severity measures for acute asthma outcomes: a first step in developing an asthma clinical prediction rule. Am J Emerg Med 2008; 26: 473-479.

55 Steele DW, Santucci KA, Wright RO, et al. Pulsus paradoxus: an objective measure of severity in croup. Am J Respir Crit Care Med 1998; 157: 331-334.

56 Shiomi T, Guilleminault C, Stoohs R, et al. Leftward shift of the interventricular septum and pulsus paradoxus in obstructive sleep apnea syndrome. Chest 1991; 100: 894-902.

57 Michard F, Boussat S, Chemla D, et al. Relation between respiratory changes in arterial pulse pressure and fluid responsiveness in septic patients with acute circulatory failure. Am J Respir Crit Care Med 2000; 162: 134-138.

58 Marik PE, Cavallazzi R, Vasu T, et al. Dynamic changes in arterial waveform derived variables and fluid responsiveness in mechanically ventilated patients: a systematic review of the literature. Crit Care Med 2009; 37: 2642-2647.

59 Marik PE, Monnet X, Teboul JL. Hemodynamic parameters to guide fluid therapy. Ann Intensive Care 2011; 1: 1. 